Теорія Ймовір. та Матем. Статист. Вип. 77, 2007
Theor. Probability and Math. Statist.

No. 77, 2008, Pages 91-105 S 0094-9000(09)00749-2

Article electronically published on January 16, 2009

\title{
A METHOD OF MODELLING LOG GAUSSIAN COX PROCESSES
} UDC 519.21

\author{
YU. V. KOZACHENKO AND O. O. POGORILYAK
}

\begin{abstract}
We consider a method for constructing models of log Gaussian Cox processes with random intensity. Namely, we consider Cox processes whose intensities are generated by a log Gaussian process. The models are constructed with a given accuracy and reliability.
\end{abstract}

\section{INTRODUCTION}

Cox processes are a natural generalization of Poisson processes for which intensities are random measures. In particular, an integral of a nonnegative stochastic process $Z(\omega, t)$ is an example of a random measure $\mu(\cdot)$. Given a realization of $Z(\omega, t)$, the corresponding process is Poissonian with intensity $\mu(B)=\int_{B} Z\left(\omega_{0}, t\right) d t$.

Some problems of actuarial mathematics require a consideration of Cox processes, since the Poisson process not always adequately models the flow of incoming claims. Cox processes serve also as models for allocations of some point processes arising in natural sciences (see [1, 2]).

Some properties of log Gaussian Cox processes are studied in [3, 4, 5]. A method of constructing models for these processes is also discussed in those papers. The aim of the current paper is to present a new method of constructing models for log Gaussian Cox processes. In contrast to the method discussed in [3, 4, 5], we construct models with a given accuracy and reliability. A similar approach for modelling stochastic processes is developed in 6, 7, 8,

Let $\{\Omega, \mathfrak{F}, \mathrm{P}\}$ be a standard probability space, and let $\{Y(t), t \in \mathbf{T}\}$ be a mean square continuous stationary Gaussian stochastic process such that $\mathrm{E} Y(t)=0$ and $\mathrm{E} Y(t) Y(s)=$ $B(t-s)$.

Definition 1.1. Let $Z(t)$ be a nonnegative stochastic process. If the conditional distribution of the process $\{\nu(B), B \in \mathfrak{B}\}$, given an arbitrary realization of $Z(t)$, is Poissonian with the intensity function

$$
\mu(B)=\int_{B} Z\left(\omega_{0}, t\right) d t
$$

then $\nu(B)$ is called a stochastic Cox process governed by $Z(t)$. If $Z(t)=\exp \{Y(t)\}$, then $\nu(B)$ is called a log Gaussian Cox process or a Cox process governed by a log Gaussian process $\exp \{Y(t)\}$.

2000 Mathematics Subject Classification. Primary 68U20; Secondary 60G10.

Key words and phrases. Log Gaussian Cox processes, random intensity, models of stochastic processes, accuracy, reliability. 
In what follows we consider the log Gaussian Cox processes. Note that the intensity of such processes is given by

$$
\mu(B)=\int_{B} \exp \{Y(t, \cdot)\} d t
$$

where $Y(t, \cdot), t \in \mathbf{T}$, is a realization of the process $\{Y(t), t \in \mathbf{T}\}$.

Consider a measurable space $\{\mathbf{T}, \mathfrak{B}\}$ with a finite measure $\mu(\cdot)$.

Definition 1.2. A point process $N$ is called a Poissonian ensemble if the random variables $N\left(B_{k}\right), k=1, \ldots, m$, are jointly independent and have the Poisson distribution with parameter $\mu\left(B_{k}\right)$, that is,

$$
\mathrm{P}\left\{N\left(B_{k}\right)=l\right\}=\frac{\left(\mu\left(B_{k}\right)\right)^{l}}{l !} \exp \left\{-\mu\left(B_{k}\right)\right\}
$$

for all $B_{k} \in \mathfrak{B}, k=1, \ldots, m, m \in \mathbf{N}$, such that $B_{i} \cap B_{j}=\varnothing$ if $i \neq j$.

Let $\xi_{i}, i=1,2, \ldots$, be independent identically distributed random elements such that

$$
\mathrm{P}\left\{\xi_{i} \in B\right\}=\frac{\mu(B)}{\mu(\mathbf{T})}
$$

for all sets $B \in \mathfrak{B}$.

Let $\Theta$ be a Poisson random variable that does not depend on $\xi_{i}$. Consider a family of random elements $\xi_{1}, \xi_{2}, \ldots, \xi_{\Theta}$. Denote by $\Pi(B)$ the number of elements of the family $\xi_{1}, \xi_{2}, \ldots, \xi_{\Theta}$ that belong to $B \in \mathfrak{B}$.

Theorem 1.1. $\Pi(B), B \in \mathfrak{B}$, is a Poisson ensemble with intensity $\mu(B)$.

Proof. Let $B_{1}, B_{2}, \ldots, B_{m} \in \mathfrak{B}$. Since the joint distribution of the random variables $\Pi\left(B_{1}\right), \Pi\left(B_{2}\right), \ldots, \Pi\left(B_{m}\right)$ given $\Theta=n$ is polynomial, the law of total probability implies that

$$
\begin{aligned}
& \mathrm{P}\{\Pi\left.\left(B_{1}\right)=k_{1}, \Pi\left(B_{2}\right)=k_{2}, \ldots, \Pi\left(B_{m}\right)=k_{m}\right\} \\
&= \sum_{n=\sum_{i=1}^{m} k_{i}}^{\infty} \mathrm{P}\left\{\Pi\left(B_{1}\right)=k_{1}, \Pi\left(B_{2}\right)=k_{2}, \ldots, \Pi\left(B_{m}\right)=k_{m} / \Theta=n\right\} \mathrm{P}\{\Theta=n\} \\
&= \sum_{n=\sum_{i=1}^{m} k_{i}}^{\infty} \frac{n !}{k_{1} ! \cdots k_{m} !\left(n-\sum_{i=1}^{m} k_{i}\right) !} \\
& \times \prod_{i=1}^{m}\left(\frac{\mu\left(B_{i}\right)}{\mu(\mathbf{T})}\right)^{k_{i}}\left(1-\frac{\sum_{i=1}^{m} \mu\left(B_{i}\right)}{\mu(\mathbf{T})}\right)^{n-\sum_{i=1}^{m} k_{i}} e^{-\mu(\mathbf{T})} \frac{(\mu(\mathbf{T}))^{n}}{n !} \\
&= \prod_{i=1}^{m} \frac{\left(\mu\left(B_{i}\right)\right)^{k_{i}}}{k_{i} !(\mu(\mathbf{T}))^{k_{i}}} e^{-\mu(\mathbf{T})} \\
& \quad \times \sum_{n=\sum_{i=1}^{m} k_{i}}^{\infty} \frac{1}{\left(n-\sum_{i=1}^{m} k_{i}\right) !} \frac{\left(\mu(\mathbf{T})-\sum_{i=1}^{m} \mu\left(B_{i}\right)\right)^{n-\sum_{i=1}^{m} k_{i}}}{(\mu(\mathbf{T}))^{n-\sum_{i=1}^{m} k_{i}}(\mu(\mathbf{T}))^{n}} \\
&=\prod_{i=1}^{m} \frac{\left(\mu\left(B_{i}\right)\right)^{k_{i}}}{k_{i} !} e^{-\mu(\mathbf{T})} \sum_{n=\sum_{i=1}^{m} k_{i}}^{\infty} \frac{1}{\left(n-\sum_{i=1}^{m} k_{i}\right) !}\left(\mu(\mathbf{T})-\sum_{i=1}^{m} \mu\left(B_{i}\right)\right)^{n-\sum_{i=1}^{m} k_{i}} \\
&=\prod_{i=1}^{m} \frac{\left(\mu\left(B_{i}\right)\right)^{k_{i}}}{k_{i} !} e^{-\mu(\mathbf{T})} \sum_{s=0}^{\infty} \frac{1}{s !}\left(\mu(\mathbf{T})-\sum_{i=1}^{m} \mu\left(B_{i}\right)\right)^{s} \\
&=\prod_{i=1}^{m} \exp \left\{-\mu\left(B_{i}\right)\right\} \frac{\left(\mu\left(B_{i}\right)\right)^{k_{i}}}{k_{i} !}
\end{aligned}
$$


Thus the $\log$ Gaussian Cox process $\nu(B)$ is a Poisson ensemble given a fixed realization of the process $Y(t)$.

\section{Constructing a model of a log Gaussian Cox process with a given ACCURACY AND RELIABILITY}

Let $\mathbf{T}=[0, T]$ for $T \in \mathbf{R}$. The procedure of constructing a model of a log Gaussian Cox process consists of three steps. First, we model a Gaussian stochastic process $Y(t)$, and then, a Poisson random variable with intensity

$$
\widetilde{\mu}(\mathbf{T})=\int_{0}^{T} \exp \{\widetilde{Y}(t)\} d t
$$

where $\widetilde{Y}(t)$ is a model of $Y(t)$. As a result, we obtain the value $\widetilde{\nu}(\mathbf{T})$. According to Theorem 1.1 a model for $\widetilde{\nu}(\mathbf{T})$ independent random variables with the distribution function

$$
\widetilde{F}(x)=\frac{\int_{0}^{x} \exp \{\widetilde{Y}(t)\} d t}{\int_{T} \exp \{\widetilde{Y}(u)\} d u}
$$

can be used to construct a model of $\log$ Gaussian Cox process. Since $F^{-1}(\zeta)$ is a model for a continuous random variable with the distribution function $F(x)$, where $\zeta$ is a uniform random variable on the interval $[0,1]$, and since a model of the log Gaussian Cox process should be as close to the process as possible, a model of the process is "acceptable" if the difference $\left|F^{(-1)}(\zeta)-\widetilde{F}^{(-1)}(\zeta)\right|$ is as small as possible for all values of $\zeta$. In other words, the position of every point of the model must be almost the same as that of the original log Gaussian Cox process if the model is "acceptable".

Definition 2.1. We say that a model $\{\widetilde{\nu}(B), B \in \mathfrak{B}\}$ approximates a log Gaussian Cox process with accuracy $\alpha, 0<\alpha<1$, and reliability $1-\gamma, 0<\gamma<1$, if

$$
\mathrm{P}\left\{\sup _{0 \leq \zeta \leq 1}\left|F^{(-1)}(\zeta)-\widetilde{F}^{(-1)}(\zeta)\right|>\alpha\right\}<\gamma .
$$

Let $X=\{X(t), t \in \mathbf{T}\}$ be a stochastic process belonging to the space $\mathrm{L}_{p}(\Omega), p \geq 1$ (that is, $\mathrm{E}|X(t)|^{p}<\infty$ for all $t \in T$ ), such that $\sup _{t \in \mathbf{T}}\|X(t)\|_{\mathrm{L}_{p}}<+\infty$. Also let

$$
\rho_{X}(t, s)=\|X(t)-X(s)\|_{\mathrm{L}_{p}}
$$

be the pseudometric generated by the process $X$, and let $N(\varepsilon)$ be the metric capacity of the pseudometric space $\left(\mathbf{T}, \rho_{X}\right)$, that is, $N(\varepsilon)$ is the number of elements of a minimal $\varepsilon$-covering of $\left(\mathbf{T}, \rho_{X}\right)$. Put $\varepsilon_{0}=\sup _{t, s \in \mathbf{T}} \rho_{X}(t, s)$.

Theorem $2.1([9])$. Suppose a stochastic process $X=\{X(t), t \in \mathbf{T}\}$ is separable in the space $\left(\mathbf{T}, \rho_{X}\right)$ and

$$
\int_{0}^{\varepsilon_{0}} N^{1 / p}(\varepsilon) d \varepsilon<+\infty
$$

Then

$$
\left(\mathrm{E}\left[\sup _{t \in \mathbf{T}}|X(t)|\right]^{p}\right)^{1 / p} \leq V_{p}
$$

where

and

$$
V_{p}=\inf _{t \in \mathbf{T}}\left(\mathrm{E}|X(t)|^{p}\right)^{1 / p}+\inf _{0<\theta<1} \frac{1}{\theta(1-\theta)} \int_{0}^{\theta \varepsilon_{0}} N^{1 / p}(\varepsilon) d \varepsilon
$$

for all $x>0$.

$$
\mathrm{P}\left\{\sup _{t \in \mathbf{T}}|X(t)| \geq x\right\} \leq \frac{V_{p}^{p}}{x^{p}}
$$


Corollary 2.1. Let $X=\{X(t), t \in \mathbf{T}\}, \mathbf{T}=[0, T], T>0$, be a separable stochastic process belonging to the space $\mathrm{L}_{p}$. Assume that there exists an increasing and continuous function $\varphi=\{\varphi(\varepsilon), \varepsilon>0\}$ such that $\varphi(0)=0$ and $\sup _{t \in \mathbf{T}}\|X(t+\varepsilon)-X(t)\|_{\mathrm{L}_{p}} \leq \varphi(\varepsilon)$. If

$$
\int_{0}^{\theta \varepsilon_{0}}\left(\varphi^{(-1)}(\varepsilon)\right)^{-1 / p} d \varepsilon<\infty
$$

where $\varphi^{(-1)}(\varepsilon)$ is the inverse function to $\varphi(\varepsilon)$ for $\varepsilon>0$, then

for all $x>0$. Here

$$
\mathrm{P}\left\{\sup _{t \in \mathbf{T}}|X(t)| \geq x\right\} \leq \frac{V_{p}^{p}}{x^{p}}
$$

$$
V_{p}=\inf _{t \in \mathbf{T}}\left(\mathrm{E}|X(t)|^{p}\right)^{1 / p}+\inf _{0<\theta<\frac{\varphi(T / 2)}{\varepsilon_{0}}} \frac{1}{\theta(1-\theta)} \int_{0}^{\theta \varepsilon_{0}}\left(\frac{T}{\varphi^{(-1)}(\varepsilon)}\right)^{1 / p} d \varepsilon .
$$

Corollary 2.1 follows from the fact that $X$ is a separable process on the interval $[0, T]$ equipped with the usual metric. This implies that the process $X(t)$ is separable in the space $\left([0, T], \rho_{X}\right)$ (see [9]) and, moreover,

$$
\frac{2 \varphi^{(-1)}\left(\theta \varepsilon_{0}\right)}{T} \leq 1
$$

for $\theta<\varphi(T / 2) / \varepsilon_{0}$. Thus ( 9 ])

$$
N(\varepsilon) \leq \frac{T}{2 \varphi^{(-1)}(\varepsilon)}+1 \leq \frac{T}{\varphi^{(-1)}(\varepsilon)} .
$$

Since $Y(t)$ is a stationary Gaussian and mean square continuous stochastic process, the following representation is well known:

$$
Y(t)=\int_{0}^{\infty} \cos \lambda t d \xi(\lambda)+\int_{0}^{\infty} \sin \lambda t d \eta(\lambda)
$$

(see [10]), where $\xi(\lambda)$ and $\eta(\lambda)$ are centered and independent Gaussian processes with independent increments such that

$$
\mathrm{E}\left(\xi\left(\lambda_{2}\right)-\xi\left(\lambda_{1}\right)\right)^{2}=\mathrm{E}\left(\eta\left(\lambda_{2}\right)-\eta\left(\lambda_{1}\right)\right)^{2}=F\left(\lambda_{2}\right)-F\left(\lambda_{1}\right)
$$

for all $\lambda_{1}<\lambda_{2}$. Here $F(\lambda)$ stands for the spectral function.

The sum

$$
\tilde{Y}(t)=\sum_{k=0}^{N-1}\left(\cos \lambda_{k} t \Delta_{k} \xi(\lambda)+\sin \lambda_{k} t \Delta_{k} \eta(\lambda)\right)
$$

is called a model of the process $Y$; here

$$
\Delta_{k} \xi(\lambda)=\int_{\lambda_{k}}^{\lambda_{k+1}} d \xi(\lambda), \quad \Delta_{k} \eta(\lambda)=\int_{\lambda_{k}}^{\lambda_{k+1}} d \eta(\lambda)
$$

and the $\lambda_{k}$ are the points of the partition $D_{\Lambda}$, namely $0=\lambda_{0}<\lambda_{1}<\cdots<\lambda_{N}=\Lambda$.

Lemma 2.1. Let $Y(t)$ be a stationary centered and mean square continuous Gaussian stochastic process with the spectral function $F(\lambda)$. Assume that a partition $D_{\Lambda}$ of the interval $[0, \Lambda], \Lambda \in \mathbf{R}$, is such that $\lambda_{k-1}-\lambda_{k}=\Lambda / N, N \in \mathbf{N}$. Then for all $p>1$,

$$
\begin{aligned}
\left(\mathrm{E}\left|\int_{0}^{T} e^{Y(u)-Y(t)} d u-\int_{0}^{T} e^{\tilde{Y}(u)-\tilde{Y}(t)} d u\right|^{p}\right)^{1 / p} & \\
\leq & 2^{1 / p} \sqrt{v_{1}} A_{N, t} p^{1 / 2} \exp \left\{2 p v_{2} B(0)-\frac{1}{2}\right\}
\end{aligned}
$$


where

$$
A_{N, t}=\left(\frac{T^{2}}{2}+T t\right) \sqrt{\frac{F(\Lambda)}{2}} \frac{\Lambda}{N}+2 T \sqrt{2(F(\infty)-F(\Lambda))}
$$

and where $v_{1}$ and $v_{2}$ are positive numbers such that $v_{1}^{-1}+v_{2}^{-1}=1$.

Proof. By the generalized Minkowski inequality,

$$
\begin{aligned}
\left(\mathrm{E}\left|\int_{0}^{T} e^{Y(u)-Y(t)} d u-\int_{0}^{T} e^{\tilde{Y}(u)-\tilde{Y}(t)} d u\right|^{p}\right)^{1 / p} & \\
\leq & \int_{0}^{T}\left(\mathrm{E}\left|e^{Y(u)-Y(t)}-e^{\tilde{Y}(u)-\tilde{Y}(t)}\right|^{p}\right)^{1 / p} d u
\end{aligned}
$$

We apply the inequality $\left|e^{x}-e^{y}\right| \leq|x-y| e^{\max (x, y)}$ and then the Hölder inequality:

$$
\begin{aligned}
\mathrm{E}\left|e^{Y(u)-Y(t)}-e^{\tilde{Y}(u)-\tilde{Y}(t)}\right|^{p} \\
\leq \mathrm{E}\left(|Y(u)-Y(t)-\tilde{Y}(u)+\widetilde{Y}(t)|^{p} e^{p \max (Y(u)-Y(t), \tilde{Y}(u)-\tilde{Y}(t))}\right) \\
\leq\left(\mathrm{E}|Y(u)-Y(t)-\tilde{Y}(u)+\tilde{Y}(t)|^{p v_{1}}\right)^{1 / v_{1}} \\
\quad \times\left(\mathrm{E} e^{p v_{2} \max (Y(u)-Y(t), \tilde{Y}(u)-\tilde{Y}(t))}\right)^{1 / v_{2}}
\end{aligned}
$$

recalling that $v_{1}^{-1}+v_{2}^{-1}=1$.

All factors on the right-hand side of the latter inequality are estimated separately. Note that

$$
\mathrm{E}|\xi|^{p}=c_{p}\left(\sigma^{2}\right)^{p / 2}
$$

for a centered Gaussian random variable $\xi$ with the variance $\sigma^{2}$. Moreover

$$
c_{p} \leq \sqrt{2} p^{p / 2} \exp \left\{-\frac{p}{2}\right\} .
$$

Therefore

$$
\mathrm{E}|Y(u)-Y(t)-\tilde{Y}(u)+\tilde{Y}(t)|^{p v_{1}}=c_{p v_{1}}\left(\mathrm{E}|Y(u)-Y(t)-\tilde{Y}(u)+\tilde{Y}(t)|^{2}\right)^{p v_{1} / 2}
$$

Considering representation (10) of the process $Y(t)$, we obtain

$$
\begin{aligned}
\mathrm{E} \mid Y(u) & -Y(t)-\tilde{Y}(u)+\left.\tilde{Y}(t)\right|^{2} \\
= & \sum_{k=0}^{N-1} \int_{\lambda_{k}}^{\lambda_{k+1}}\left(\cos \lambda u-\cos \lambda_{k} u-\cos \lambda t+\cos \lambda_{k} t\right)^{2} d F(\lambda) \\
& +\sum_{k=0}^{N-1} \int_{\lambda_{k}}^{\lambda_{k+1}}\left(\sin \lambda u-\sin \lambda_{k} u-\sin \lambda t+\sin \lambda_{k} t\right)^{2} d F(\lambda) \\
& +\int_{\Lambda}^{\infty}(\cos \lambda u-\cos \lambda t)^{2} d F(\lambda)+\int_{\Lambda}^{\infty}(\sin \lambda u-\sin \lambda t)^{2} d F(\lambda)
\end{aligned}
$$




$$
\begin{aligned}
& \leq 4 \sum_{k=0}^{N-1} \int_{\lambda_{k}}^{\lambda_{k+1}}\left(\left|\sin \frac{u\left(\lambda+\lambda_{k}\right)}{2} \sin \frac{u\left(\lambda-\lambda_{k}\right)}{2}\right|\right. \\
& \left.\quad+\left|\sin \frac{t\left(\lambda_{k}+\lambda\right)}{2} \sin \frac{t\left(\lambda-\lambda_{k}\right)}{2}\right|\right)^{2} d F(\lambda) \\
& \quad+4 \sum_{k=0}^{N-1} \int_{\lambda_{k}}^{\lambda_{k+1}}\left(\left|\sin \frac{u\left(\lambda-\lambda_{k}\right)}{2} \cos \frac{u\left(\lambda+\lambda_{k}\right)}{2}\right|\right. \\
& \left.\quad+\left|\sin \frac{t\left(\lambda-\lambda_{k}\right)}{2} \sin \frac{t\left(\lambda_{k}+\lambda\right)}{2}\right|\right)^{2} d F(\lambda) \\
& \quad+8(F(\infty)-F(\Lambda)) \\
& \leq 2 \frac{\Lambda^{2}}{N^{2}}(u+t)^{2} F(\Lambda)+8(F(\infty)-F(\Lambda)) .
\end{aligned}
$$

Hence

$$
\left(\mathrm{E}|Y(u)-Y(t)-\tilde{Y}(u)+\tilde{Y}(t)|^{p v_{1}}\right)^{1 / v_{1}} \leq c_{p v_{1}}^{1 / v_{1}} A_{N, u, t}^{p / 2},
$$

where

$$
A_{N, u, t}=2 \frac{\Lambda^{2}}{N^{2}}(u+t)^{2} F(\Lambda)+8(F(\infty)-F(\Lambda)) .
$$

Now we estimate $\operatorname{E} \exp \left\{p v_{2} \max (Y(u)-Y(t), \tilde{Y}(u)-\tilde{Y}(t))\right\}$. Note that

$$
\mathrm{E} \exp \{\lambda \xi\}=\exp \left\{\lambda^{2} \mathrm{E} \xi^{2} / 2\right\}
$$

for a Gaussian random variable $\xi$. Using this result we get

$$
\begin{gathered}
\mathrm{E} e^{p v_{2} \max (Y(u)-Y(t), \widetilde{Y}(u)-\tilde{Y}(t))} \leq \mathrm{E} e^{p v_{2}(Y(u)-Y(t))}+\mathrm{E} e^{p v_{2}(\widetilde{Y}(u)-\widetilde{Y}(t))} \\
=\exp \left\{\frac{\left(p v_{2}\right)^{2}}{2} \mathrm{E}(Y(u)-Y(t))^{2}\right\} \\
+\left\{\frac{\left(p v_{2}\right)^{2}}{2} \mathrm{E}(\widetilde{Y}(u)-\widetilde{Y}(t))^{2}\right\} .
\end{gathered}
$$

It is easy to see that

$$
\mathrm{E}|Y(u)-Y(t)|^{2} \leq 4 B(0) \quad \text { and } \quad \mathrm{E}|\tilde{Y}(u)-\tilde{Y}(t)|^{2} \leq 4 B(0) .
$$

Then

$$
\mathrm{E} e^{p v_{2} \max (Y(u)-Y(t), \widetilde{Y}(u)-\widetilde{Y}(t))} \leq 2 e^{2\left(p v_{2}\right)^{2} B(0)} .
$$

Applying bound (6) and the latter inequality, we derive from (3) that

$$
\mathrm{E}\left|e^{Y(u)-Y(t)}-e^{\tilde{Y}(u)-\tilde{Y}(t)}\right|^{p} \leq c_{p v_{1}}^{1 / v_{1}} A_{N, u, t^{p}}^{p / 2} 2^{1 / v_{2}} e^{2 p^{2} v_{2} B(0)},
$$

where $A_{N, u, t}$ is defined by (7). Taking into account the bound for $c_{p}$ given in (5), we have

$$
\begin{aligned}
\int_{0}^{T} & \left(\mathrm{E}\left|e^{Y(u)-Y(t)}-e^{\widetilde{Y}(u)-\widetilde{Y}(t)}\right|^{p}\right)^{1 / p} d u \\
& \leq \int_{0}^{T}\left(\sqrt{2}\left(p v_{1}\right)^{p v_{1} / 2} \exp \left\{-\frac{p v_{1}}{2}\right\}\right)^{1 / p v_{1}} A_{N, u, t^{1 / p v_{2}}}^{1 / 2} e^{2 p v_{2} B(0)} d u
\end{aligned}
$$

The integrand is estimated by an elementary inequality $\sqrt{x+y} \leq \sqrt{x}+\sqrt{y}$. Integrating and performing some easy algebraic transformations, we obtain Lemma 2.1 from inequality (2). 
Lemma 2.2. Let $Y(t)$ be a centered mean square continuous stationary Gaussian process with the spectral function $F(\lambda)$. Assume that the spectral moment

$$
\int_{0}^{\infty} \lambda^{2 \beta} d F(\lambda), \quad 0<\beta \leq 1,
$$

exists. Let a partition $D_{\Lambda}$ of the interval $[0, \Lambda], \Lambda \in \mathbf{R}$, be such that

$$
\lambda_{k-1}-\lambda_{k}=\Lambda / N, \quad N \in \mathbf{N} .
$$

Then for all $p>1$,

$$
\begin{aligned}
\left(\mathrm{E} \mid \int_{0}^{T} e^{Y(u)-Y(t+h)} d u\right. & -\int_{0}^{T} e^{\tilde{Y}(u)-\widetilde{Y}(t+h)} d u \\
& \left.-\int_{0}^{T} e^{Y(u)-Y(t)} d u+\left.\int_{0}^{T} e^{\widetilde{Y}(u)-\widetilde{Y}(t)} d u\right|^{p}\right)^{1 / p} \leq h G_{p, t},
\end{aligned}
$$

where

$$
\begin{gathered}
G_{p, t}=2^{1 /(2 p)}\left(T \sqrt{2 P_{N}}+\left(2^{7-2 \beta} \Lambda^{2 \beta} B(0)\right)^{1 / 2} A_{N, t}\right) p e^{16 p B(0)-1 / 2} \\
P_{N}=2^{5-4 \beta}\left(\left(\frac{\Lambda}{N}\right)^{\beta}+2^{-\beta-1} T \frac{\Lambda^{\beta+1}}{N}\right)^{2} F(\Lambda)+2^{3-2 \beta} \int_{\Lambda}^{\infty} \lambda^{2 \beta} d F(\lambda), \\
A_{N, t}=\left(\frac{T^{2}}{2}+T t\right) \sqrt{\frac{F(\Lambda)}{2}} \frac{\Lambda}{N}+2 T \sqrt{2(F(\infty)-F(\Lambda))} .
\end{gathered}
$$

Proof. We have

$$
\begin{gathered}
\text { (E| } \int_{0}^{T} e^{Y(u)-Y(t+h)} d u-\int_{0}^{T} e^{\tilde{Y}(u)-\tilde{Y}(t+h)} d u \\
\left.-\int_{0}^{T} e^{Y(u)-Y(t)} d u+\left.\int_{0}^{T} e^{\tilde{Y}(u)-\tilde{Y}(t)} d u\right|^{p}\right)^{1 / p} \\
=\left(\mathrm{E} \mid \int_{0}^{T} e^{Y(u)-Y(t)} d u \cdot e^{Y(t)-Y(t+h)}-\int_{0}^{T} e^{Y(u)-Y(t)} d u \cdot e^{\tilde{Y}(t)-\tilde{Y}(t+h)}\right. \\
+\int_{0}^{T} e^{Y(u)-Y(t)} d u \cdot e^{\tilde{Y}(t)-\tilde{Y}(t+h)}-\int_{0}^{T} e^{Y(u)-Y(t)} d u \\
\left.-\int_{0}^{T} e^{\tilde{Y}(u)-\tilde{Y}(t)} d u \cdot e^{\tilde{Y}(t)-\tilde{Y}(t+h)}+\left.\int_{0}^{T} e^{\tilde{Y}(u)-\tilde{Y}(t)} d u\right|^{p}\right)^{1 / p} \\
=\left(\mathrm{E} \mid \int_{0}^{T} e^{Y(u)-Y(t)} d u\left(e^{Y(t)-Y(t+h)}-e^{\tilde{Y}(t)-\tilde{Y}(t+h)}\right)\right. \\
+\int_{0}^{T} e^{Y(u)-Y(t)} d u\left(e^{\tilde{Y}(t)-\tilde{Y}(t+h)}-1\right) \\
\left.-\left.\int_{0}^{T} e^{\tilde{Y}(u)-\tilde{Y}(t)} d u\left(e^{\tilde{Y}(t)-\tilde{Y}(t+h)}-1\right)\right|^{p}\right)^{1 / p}
\end{gathered}
$$




$$
\begin{aligned}
\leq\left(\mathrm{E}\left|\int_{0}^{T} e^{Y(u)-Y(t)} d u\left(e^{Y(t)-Y(t+h)}-e^{\tilde{Y}(t)-\tilde{Y}(t+h)}\right)\right|^{p}\right)^{1 / p} \\
\quad+\left(\mathrm{E}\left|\left(e^{\tilde{Y}(t)-\tilde{Y}(t+h)}-1\right) \int_{0}^{T}\left(e^{Y(u)-Y(t)}-e^{\tilde{Y}(u)-\tilde{Y}(t)}\right) d u\right|^{p}\right)^{1 / p} .
\end{aligned}
$$

Every term on the right-hand side of (8) is estimated separately. For the first term we apply the generalized Minkowski inequality together with the estimate

$$
\left|e^{x}-e^{y}\right| \leq|x-y| e^{\max (x, y)},
$$

and Hölder's inequality:

$$
\begin{aligned}
& \left(\mathrm{E}\left|\int_{0}^{T} e^{Y(u)-Y(t)} d u\left(e^{Y(t)-Y(t+h)}-e^{\tilde{Y}(t)-\tilde{Y}(t+h)}\right)\right|^{p}\right)^{1 / p} \\
& \quad \leq \int_{0}^{T}\left(\mathrm{E}\left|e^{Y(u)-Y(t)} d u\left(e^{Y(t)-Y(t+h)}-e^{\tilde{Y}(t)-\widetilde{Y}(t+h)}\right)\right|^{p}\right)^{1 / p} d u \\
& \quad \leq \int_{0}^{T}\left(\mathrm{E}\left|e^{Y(u)-Y(t)}\right| Y(t)-Y(t+h)-\tilde{Y}(t)\right. \\
& \left.\quad+\tilde{Y}(t+h)\left|e^{\max (Y(t)-Y(t+h), \tilde{Y}(t)-\tilde{Y}(t+h))}\right|^{p}\right)^{1 / p} d u \\
& \quad \leq \int_{0}^{T}\left(\left(\mathrm{E}\left|\Delta_{1}(Y)\right|^{p r_{1}}\right)^{1 / r_{1}}\left(\mathrm{E}\left|\Delta_{2}(Y)\right|^{p r_{2}}\right)^{1 / r_{2}}\right)^{1 / p} d u,
\end{aligned}
$$

where

$$
\begin{gathered}
\Delta_{1}(Y)=Y(t)-Y(t+h)-\tilde{Y}(t)+\tilde{Y}(t+h), \\
\Delta_{2}(Y)=e^{Y(u)-Y(t)} e^{\max (Y(t)-Y(t+h), \tilde{Y}(t)-\tilde{Y}(t+h))},
\end{gathered}
$$

and

$$
r_{1}^{-1}+r_{2}^{-1}=1
$$

By equality (4),

$$
\left(\mathrm{E}\left|\Delta_{1}(Y)\right|^{p r_{1}}\right)=\left(\mathrm{E}\left|\Delta_{1}(Y)\right|^{2}\right)^{p r_{1} / 2} c_{p r_{1}} .
$$

Using representation (11) for the process $\tilde{Y}(t)$, we get

$$
\begin{aligned}
& \mathrm{E}\left|\Delta_{1}(Y)\right|^{2}=\mathrm{E}|Y(t+h)-\tilde{Y}(t+h)-Y(t)+\tilde{Y}(t)|^{2} \\
& =\sum_{k=0}^{N-1} \int_{\lambda_{k}}^{\lambda_{k+1}}\left(\cos \lambda(t+h)-\cos \lambda_{k}(t+h)-\cos \lambda t+\cos \lambda_{k} t\right)^{2} d F(\lambda) \\
& \quad+\sum_{k=0}^{N-1} \int_{\lambda_{k}}^{\lambda_{k+1}}\left(\sin \lambda(t+h)-\sin \lambda_{k}(t+h)-\sin \lambda t+\sin \lambda_{k} t\right)^{2} d F(\lambda) \\
& \quad+\int_{\Lambda}^{\infty}(\cos \lambda(t+h)-\cos \lambda t)^{2} d F(\lambda)+\int_{\Lambda}^{\infty}(\sin \lambda(t+h)-\sin \lambda t)^{2} d F(\lambda)
\end{aligned}
$$




$$
\begin{aligned}
& \leq \sum_{k=0}^{N-1} \int_{\lambda_{k}}^{\lambda_{k+1}} 4\left(\left|\sin \frac{(t+h)\left(\lambda+\lambda_{k}\right)}{2}\right|\left|\sin \frac{(t+h)\left(\lambda-\lambda_{k}\right)}{2}-\sin \frac{\left(\lambda-\lambda_{k}\right) t}{2}\right|\right. \\
& \left.+\left|\sin \frac{\left(\lambda-\lambda_{k}\right) t}{2}\right|\left|\sin \frac{(t+h)\left(\lambda+\lambda_{k}\right)}{2}-\sin \frac{\left(\lambda+\lambda_{k}\right) t}{2}\right|\right)^{2} d F(\lambda) \\
& +\sum_{k=0}^{N-1} \int_{\lambda_{k}}^{\lambda_{k+1}} 4\left(\left|\sin \frac{(t+h)\left(\lambda-\lambda_{k}\right)}{2}\right|\left|\cos \frac{(t+h)\left(\lambda+\lambda_{k}\right)}{2}-\cos \frac{\left(\lambda+\lambda_{k}\right) t}{2}\right|\right. \\
& \left.+\left|\cos \frac{\left(\lambda+\lambda_{k}\right) t}{2}\right|\left|\sin \frac{(t+h)\left(\lambda-\lambda_{k}\right)}{2}-\sin \frac{\left(\lambda-\lambda_{k}\right) t}{2}\right|\right)^{2} d F(\lambda) \\
& +\int_{\Lambda}^{\infty} 4\left|\sin \frac{\lambda(2 t+h)}{2}\right|^{2}\left|\sin \frac{\lambda h}{2}\right|^{2} d F(\lambda) \\
& +\int_{\Lambda}^{\infty} 4\left|\cos \frac{\lambda(2 t+h)}{2}\right|^{2}\left|\sin \frac{\lambda h}{2}\right|^{2} d F(\lambda) \\
& \leq \sum_{k=0}^{N-1} \int_{\lambda_{k}}^{\lambda_{k+1}} 4\left(\left|2 \cos \frac{(2 t+h)\left(\lambda-\lambda_{k}\right)}{4} \sin \frac{\left(\lambda-\lambda_{k}\right) h}{4}\right|\right. \\
& \left.+\left|\sin \frac{\left(\lambda-\lambda_{k}\right) t}{2}\right|\left|2 \cos \frac{(2 t+h)\left(\lambda+\lambda_{k}\right)}{4} \sin \frac{\left(\lambda+\lambda_{k}\right) h}{4}\right|\right)^{2} d F(\lambda) \\
& +\sum_{k=0}^{N-1} \int_{\lambda_{k}}^{\lambda_{k+1}} 4\left(\left|\sin \frac{(t+h)\left(\lambda-\lambda_{k}\right)}{2}\right|\left|2 \sin \frac{(2 t+h)\left(\lambda+\lambda_{k}\right)}{4} \sin \frac{\left(\lambda+\lambda_{k}\right) h}{4}\right|\right. \\
& \left.+\left|2 \cos \frac{(2 t+h)\left(\lambda-\lambda_{k}\right)}{4} \sin \frac{\left(\lambda-\lambda_{k}\right) h}{4}\right|\right)^{2} d F(\lambda) \\
& +2^{3-2 \beta} h^{2 \beta} \int_{\Lambda}^{\infty} \lambda^{2 \beta} d F(\lambda) \\
& \leq \sum_{k=0}^{N-1} \int_{\lambda_{k}}^{\lambda_{k+1}} 16\left(\frac{\left(\lambda-\lambda_{k}\right)^{\beta} h^{\beta}}{4^{\beta}}+\frac{\left(\lambda-\lambda_{k}\right) t}{2} \frac{\left(\lambda+\lambda_{k}\right)^{\beta} h^{\beta}}{4^{\beta}}\right)^{2} d F(\lambda) \\
& +\sum_{k=0}^{N-1} \int_{\lambda_{k}}^{\lambda_{k+1}} 16\left(\frac{(t+h)\left(\lambda-\lambda_{k}\right)}{2} \frac{\left(\lambda+\lambda_{k}\right)^{\beta} h^{\beta}}{4^{\beta}}+\frac{\left(\lambda-\lambda_{k}\right)^{\beta} h}{4^{\beta}}\right)^{2} d F(\lambda) \\
& +2^{3-2 \beta} h^{2 \beta} \int_{\Lambda}^{\infty} \lambda^{2 \beta} d F(\lambda) \\
& \leq 32 \sum_{k=0}^{N-1} \int_{\lambda_{k}}^{\lambda_{k+1}}\left(\left(\frac{\Lambda}{N} \frac{h}{4}\right)^{\beta}+\frac{\Lambda}{N} \frac{T}{2}\left(2 \Lambda \frac{h}{4}\right)^{\beta}\right)^{2} d F(\lambda)+2^{3-2 \beta} h^{2 \beta} \int_{\Lambda}^{\infty} \lambda^{2 \beta} d F(\lambda) .
\end{aligned}
$$

Then

$$
\mathrm{E}\left|\Delta_{1}(Y)\right|^{2} \leq h^{2 \beta} P_{N},
$$

where

$$
P_{N}=2^{5-4 \beta}\left(\left(\frac{\Lambda}{N}\right)^{\beta}+2^{-\beta-1} T \frac{\Lambda^{\beta+1}}{N}\right)^{2} F(\Lambda)+2^{3-2 \beta} \int_{\Lambda}^{\infty} \lambda^{2 \beta} d F(\lambda) .
$$

Therefore

$$
\left(\mathrm{E}\left|\Delta_{1}(Y)\right|^{p r_{1}}\right)^{1 / r_{1}} \leq c_{p r_{1}}^{1 / r_{1}} h^{p \beta} P_{N}^{p / 2} .
$$


Further we estimate $\mathrm{E}\left|\Delta_{2}(Y)\right|^{p r_{2}}$. By Hölder's inequality,

$$
\begin{aligned}
& \mathrm{E}\left|\Delta_{2}(Y)\right|^{p r_{2}} \\
& \quad \leq\left(\mathrm{E} e^{p r_{2} f_{1}(Y(u)-Y(t))}\right)^{1 / f_{1}}\left(\mathrm{E} e^{p r_{2} f_{2} \max (Y(t)-Y(t+h), \tilde{Y}(t)-\tilde{Y}(t+h))}\right)^{1 / f_{2}}
\end{aligned}
$$

for $f_{1}^{-1}+f_{2}^{-1}=1$. Since

$$
\mathrm{E} \exp \{\lambda \xi\}=\exp \left\{\lambda^{2} \mathrm{E} \xi^{2} / 2\right\}
$$

for a Gaussian random variable $\xi$ and since

$$
\mathrm{E}|Y(u)-Y(t)|^{2} \leq 4 B(0), \quad \mathrm{E}|\tilde{Y}(u)-\tilde{Y}(t)|^{2} \leq 4 B(0),
$$

we get

$$
\begin{aligned}
& \mathrm{E} e^{p r_{2} f_{1}(Y(u)-Y(t))}=\exp \left\{\frac{\left(p r_{2} f_{1}\right)^{2}}{2} \mathrm{E}(Y(u)-Y(u))^{2}\right\} \leq e^{2\left(p r_{2} f_{1}\right)^{2} B(0)}, \\
\mathrm{E} e^{p r_{2} f_{2} \max (Y(t)-Y(t+h), \tilde{Y}(t)-\tilde{Y}(t+h))} & \\
\leq & \exp \left\{\frac{\left(p r_{2} f_{2}\right)^{2}}{2} \mathrm{E}(Y(t)-Y(t+h))^{2}\right\}+\exp \left\{\frac{\left(p r_{2} f_{2}\right)^{2}}{2} \mathrm{E}(\tilde{Y}(t)-\tilde{Y}(t+h))^{2}\right\} \\
\leq & 2 \exp \left\{2\left(p r_{2} f_{2}\right)^{2} B(0)\right\} .
\end{aligned}
$$

Setting $f_{1}=f_{2}=2$ and combining the last two inequalities with (11), we obtain

$$
\left(\mathrm{E}\left|\Delta_{2}(Y)\right|^{p r_{2}}\right)^{1 / r_{2}} \leq 2^{1 /\left(2 r_{2}\right)} \exp \left\{8 p^{2} r_{2} B(0)\right\} .
$$

Considering inequality (10), we deduce from bound (9) that

$$
\begin{aligned}
(\mathrm{E} \mid & \left.\left.\int_{0}^{T} e^{Y(u)-Y(t)} d u\left(e^{Y(t)-Y(t+h)}-e^{\tilde{Y}(t)-\widetilde{Y}(t+h)}\right)\right|^{p}\right)^{1 / p} \\
& \leq \int_{0}^{T}\left(\left(\sqrt{2}\left(p r_{1}\right)^{p r_{1} / 2} \exp \left\{-\frac{p r_{1}}{2}\right\}\right)^{1 / r_{1}} h^{p \beta} P_{N}^{p / 2} 2^{1 /\left(2 r_{2}\right)} e^{8 p^{2} r_{2} B(0)}\right)^{1 / p} d u \\
& \leq h^{\beta} 2^{1 /(2 p)} T \sqrt{r_{1} P_{N}} p^{1 / 2} \exp \left\{8 p r_{2} B(0)-\frac{1}{2}\right\} .
\end{aligned}
$$

Now we turn to the estimation of the second term on the right-hand side of (8). It is clear that

$$
\begin{gathered}
\left(\mathrm{E}\left|\left(e^{\tilde{Y}(t)-\widetilde{Y}(t+h)}-1\right) \int_{0}^{T}\left(e^{Y(u)-Y(t)}-e^{\tilde{Y}(u)-\widetilde{Y}(t)}\right) d u\right|^{p}\right)^{1 / p} \\
\leq \int_{0}^{T}\left(\mathrm{E}\left|\left(e^{\tilde{Y}(t)-\tilde{Y}(t+h)}-1\right)\left(e^{Y(u)-Y(t)}-e^{\tilde{Y}(u)-\widetilde{Y}(t)}\right)\right|^{p}\right)^{1 / p} d u \\
\leq \int_{0}^{T}\left(\left(\mathrm{E}\left|e^{\tilde{Y}(t)-\tilde{Y}(t+h)}-1\right|^{p s_{1}}\right)^{1 / s_{1}}\right. \\
\left.\times\left(\mathrm{E}\left|\left(e^{Y(u)-Y(t)}-e^{\tilde{Y}(u)-\tilde{Y}(t)}\right)\right|^{p s_{2}}\right)^{1 / s_{2}}\right)^{1 / p} d u
\end{gathered}
$$

for $s_{1}^{-1}+s_{2}^{-1}=1$. Applying first the inequality $|\exp (x)-1| \leq|x| \exp \{|x|\}$ and then Hölder's inequality, we obtain

$$
\begin{aligned}
\mathrm{E}\left|e^{\tilde{Y}(t)-\tilde{Y}(t+h)}-1\right|^{p s_{1}} & \leq \mathrm{E}\left(|\widetilde{Y}(t)-\tilde{Y}(t+h)| e^{|\widetilde{Y}(t)-\widetilde{Y}(t+h)|}\right)^{p s_{1}} \\
& \leq \mathrm{E}\left(|\widetilde{Y}(t)-\tilde{Y}(t+h)|^{p s_{1} l_{1}}\right)^{1 / l_{1}}\left(\mathrm{E} e^{p s_{1} l_{2}|\tilde{Y}(t)-\tilde{Y}(t+h)|}\right)^{1 / l_{2}}
\end{aligned}
$$


for $l_{1}^{-1}+l_{2}^{-1}=1$. Since $\mathrm{E}|\tilde{Y}(t)-\tilde{Y}(t+h)|^{2} \leq 2^{3-2 \beta} \Lambda^{2^{\beta}} h^{2 \beta} F(\Lambda)$, we have

$$
\begin{gathered}
\left(\mathrm{E}|\widetilde{Y}(t)-\widetilde{Y}(t+h)|^{p s_{1} l_{1}}\right)^{1 / l_{1}} \leq c_{p s_{1} l_{1}}^{1 / l_{1}}\left(2^{3-2 \beta} \Lambda^{2^{\beta}} h^{2 \beta} B(0)\right)^{p s_{1} / 2} \\
\left(\mathrm{E} e^{p s_{1} l_{2}|\tilde{Y}(t)-\widetilde{Y}(t+h)|}\right)^{1 / l_{2}} \leq e^{2\left(p s_{1}\right)^{2} l_{2} B(0)}
\end{gathered}
$$

Hence

$$
\mathrm{E}\left|e^{\widetilde{Y}(t)-\tilde{Y}(t+h)}-1\right|^{p s_{1}} \leq c_{p s_{1} l_{1}}^{1 / l_{1}}\left(2^{3-2 \beta} \Lambda^{2^{\beta}} h^{2 \beta} B(0)\right)^{p s_{1} / 2} e^{2\left(p s_{1}\right)^{2} l_{2} B(0)} .
$$

Then

$$
\begin{aligned}
\mathrm{E}\left|e^{Y(u)-Y(t)}-e^{\tilde{Y}(u)-\tilde{Y}(t)}\right|^{p s_{2}} \\
\leq \mathrm{E}\left(|Y(u)-Y(t)-\tilde{Y}(u)+\widetilde{Y}(t)| e^{\max (Y(u)-Y(t), \tilde{Y}(u)-\tilde{Y}(t))}\right)^{p s_{2}} \\
\leq\left(\mathrm{E}|Y(u)-Y(t)-\tilde{Y}(u)+\tilde{Y}(t)|^{p s_{2} m_{1}}\right)^{1 / m_{1}} \\
\quad \times\left(\mathrm{E} e^{p s_{2} m_{2} \max (Y(u)-Y(t), \tilde{Y}(u)-\tilde{Y}(t))}\right)^{1 / m_{2}}
\end{aligned}
$$

for $m_{1}^{-1}+m_{2}^{-1}=1$. According to Lemma 2.1 ,

$$
\begin{gathered}
\mathrm{E}|Y(u)-Y(t)-\tilde{Y}(u)+\tilde{Y}(t)|^{2} \leq A_{N, u, t}, \\
A_{N, u, t}=2 \frac{\Lambda^{2}}{N^{2}}(u+t)^{2} F(\Lambda)+8(F(\infty)-F(\Lambda)),
\end{gathered}
$$

whence

$$
\begin{gathered}
\left(\mathrm{E}|Y(u)-Y(t)-\tilde{Y}(u)+\tilde{Y}(t)|^{p s_{2} m_{1}}\right)^{1 / m_{1}} \leq c_{p s_{2} m_{1}}^{1 / m_{1}} A_{N, u, t}^{p s_{2} / 2}, \\
\left(\mathrm{E} e^{p s_{2} m_{2} \max (Y(u)-Y(t), \tilde{Y}(u)-\tilde{Y}(t))}\right)^{1 / m_{2}} \leq 2^{1 / m_{2}} e^{2\left(p s_{2}\right)^{2} m_{2} B(0)} .
\end{gathered}
$$

Two last two inequalities imply

$$
\mathrm{E}\left|e^{Y(u)-Y(t)}-e^{\widetilde{Y}(u)-\widetilde{Y}(t)}\right|^{p s_{2}} \leq c_{p s_{2} m_{1}}^{1 / m_{1}} A_{N, u, t}^{p s_{2} / 2} 2^{1 / m_{2}} e^{2\left(p s_{2}\right)^{2} m_{2} B(0)} .
$$

Setting $s_{1}=s_{2}=l_{1}=l_{2}=m_{1}=m_{2}=2$, we derive from (14) and (13) that

$$
\begin{aligned}
& \left(\mathrm{E}\left|\left(e^{\tilde{Y}(t)-\tilde{Y}(t+h)}-1\right) \int_{0}^{T}\left(e^{Y(u)-Y(t)}-e^{\tilde{Y}(u)-\tilde{Y}(t)}\right) d u\right|^{p}\right)^{1 / p} \\
& \leq \int_{0}^{T}\left(c_{p s_{1} l_{1}}^{1 /\left(l_{1} s_{1}\right)}\left(2^{3-2 \beta} \Lambda^{2^{\beta}} h^{2 \beta} B(0)\right)^{p / 2}\right. \\
& \left.\quad \times e^{2 p^{2} s_{1} l_{2} B(0)} c_{p s_{2} m_{1}}^{1 /\left(m_{1} s_{2}\right)} A_{N, u, t}^{p / 2} 2^{1 / m_{2} s_{2}} e^{2 p^{2} s_{2} m_{2} B(0)}\right)^{1 / p} d u \\
& \leq \int_{0}^{T}\left(\left(\sqrt{2}\left(p s_{1} l_{1}\right)^{p s_{1} l_{1} / 2} e^{-p s_{1} l_{1} / 2}\right)^{1 /\left(l_{1} s_{1}\right)}\left(2^{3-2 \beta} \Lambda^{2^{\beta}} h^{2 \beta} B(0)\right)^{p / 2} e^{2 p^{2} s_{1} l_{2} B(0)}\right. \\
& \left.\quad \times\left(\sqrt{2}\left(p s_{2} m_{1}\right)^{p s_{2} m_{1} / 2} e^{-p s_{2} m_{1} / 2}\right)^{1 /\left(m_{1} s_{2}\right)} A_{N, u, t}^{p / 2} 2^{1 /\left(m_{2} s_{2}\right)} e^{2 p^{2} s_{2} m_{2} B(0)}\right)^{1 / p} d u \\
& \leq h^{\beta} \int_{0}^{T}\left(2^{1 /(2 p)} 4 A_{N, u, t}^{1 / 2}\left(2^{3-2 \beta} \Lambda^{2^{\beta}} B(0)\right)^{1 / 2} p e^{16 p B(0)-1}\right) d u .
\end{aligned}
$$


Estimating the integrand and then integrating, we get

$$
\begin{gathered}
\left(\mathrm{E}\left|\left(e^{\tilde{Y}(t)-\tilde{Y}(t+h)}-1\right) \int_{0}^{T}\left(e^{Y(u)-Y(t)}-e^{\tilde{Y}(u)-\tilde{Y}(t)}\right) d u\right|^{p}\right)^{1 / p} \\
\leq h^{\beta} 2^{1 /(2 p)} 4 A_{N, t}\left(2^{3-2 \beta} \Lambda^{2^{\beta}} B(0)\right)^{1 / 2} p e^{16 p B(0)-1}
\end{gathered}
$$

where

$$
A_{N, t}=\left(\frac{T^{2}}{2}+T t\right) \sqrt{\frac{F(\Lambda)}{2}} \frac{\Lambda}{N}+2 T \sqrt{2(F(\infty)-F(\Lambda))} .
$$

Setting $r_{1}=r_{2}=1$ and taking into account (12), we derive Lemma 2.2 from the bound (8).

Lemma 2.3. Let $Y(t)$ be a separable centered mean square continuous stationary Gaussian stochastic process with spectral function $F(\lambda)$. Assume that the spectral moment $\int_{0}^{\infty} \lambda^{2 \beta} d F(\lambda), 0<\beta \leq 1$, exists. Let a partition $D_{\Lambda}$ of the interval $[0, \Lambda], \Lambda \in \mathbf{R}$, be such that $\lambda_{k-1}-\lambda_{k}=\Lambda / N, N \in \mathbf{N}$. If $M_{N}<\alpha \exp \left\{\frac{1}{2}-32 B(0) / \beta\right\}$, then

$$
\begin{aligned}
& \mathrm{P}\left\{\sup _{0 \leq \zeta \leq 1}\left|F^{(-1)}(\zeta)-\widetilde{F}^{(-1)}(\zeta)\right|>\alpha\right\} \\
& \leq\left(\frac{L_{N}}{\alpha}\right)^{K_{N}} K_{N}^{K_{N} / 2} \exp \left\{-\frac{K_{N}}{2}+2 v_{2} K_{N}^{2}\right\} \\
&+\frac{1}{\sqrt{2}} \exp \left\{\frac{K_{N} \beta}{K_{N} \beta-1}\right\}\left(K_{N} \beta+1\right)^{K_{N}+1 / \beta} \exp \left\{-16 B(0) K_{N}^{2}\right\},
\end{aligned}
$$

where

$$
\begin{gathered}
L_{N}=2 \sqrt{v_{1}} A_{N, 0}, \quad K_{N}=\frac{\frac{1}{2}-\ln \frac{M_{N}}{\alpha}}{32 B(0)} \\
M_{N}=\frac{2 T^{\beta}}{\beta}\left(T \sqrt{2 P_{N}}+\left(2^{7-2 \beta} \Lambda^{2 \beta} B(0)\right)^{1 / 2} A_{N, T}\right), \\
A_{N, 0}=\frac{T^{2}}{2} \sqrt{\frac{F(\Lambda)}{2}} \frac{\Lambda}{N}+2 T \sqrt{2(F(\infty)-F(\Lambda))} \\
A_{N, T}=\frac{3 T^{2}}{2} \sqrt{\frac{F(\Lambda)}{2}} \frac{\Lambda}{N}+2 T \sqrt{2(F(\infty)-F(\Lambda))} \\
P_{N}=2^{5-4 \beta}\left(\left(\frac{\Lambda}{N}\right)^{\beta}+2^{-\beta-1} T \frac{\Lambda^{\beta+1}}{N}\right)^{2} F(\Lambda)+2^{3-2 \beta} \int_{\Lambda}^{\infty} \lambda^{2 \beta} d F(\lambda) .
\end{gathered}
$$

Here $B$ denotes the covariance function of the process $Y(t), v_{2}=v_{1} /\left(v_{1}-1\right)$, and $v_{1}$ is a nonnegative real number greater than 1 .

Proof. Note that

$$
\left(F^{-1}(\zeta)\right)^{\prime}=\frac{1}{F^{\prime}\left(F^{-1}(\zeta)\right)}=\int_{0}^{T} e^{Y(u)} d u \cdot e^{-Y\left(F^{-1}(\zeta)\right)} .
$$

By the Lagrange formula,

$$
\begin{aligned}
\sup _{0 \leq \zeta \leq 1}\left|F^{(-1)}(\zeta)-\widetilde{F}^{(-1)}(\zeta)\right| & \leq \sup _{0 \leq \hat{\zeta} \leq 1}\left|\left(F^{(-1)}(\hat{\zeta})\right)^{\prime}-\left(\widetilde{F}^{(-1)}(\hat{\zeta})\right)^{\prime}\right| \\
& =\sup _{0 \leq t \leq T}\left|\int_{0}^{T} e^{Y(u)-Y(t)} d u-\int_{0}^{T} e^{\tilde{Y}(u)-\tilde{Y}(t)} d u\right|
\end{aligned}
$$


Thus,

$$
\begin{aligned}
& \mathrm{P}\left\{\sup _{0 \leq \zeta \leq 1}\left|F^{(-1)}(\zeta)-\widetilde{F}^{(-1)}(\zeta)\right|>\alpha\right\} \\
& \quad \leq \mathrm{P}\left\{\sup _{0 \leq t \leq T}\left|\int_{0}^{T} e^{Y(u)-Y(t)} d u-\int_{0}^{T} e^{\tilde{Y}(u)-\tilde{Y}(t)} d u\right|>\alpha\right\} .
\end{aligned}
$$

Using Lemma 2.1, we get

$$
\begin{aligned}
\inf _{0 \leq t \leq T} & \left(\mathrm{E}\left|\int_{0}^{T} e^{Y(u)-Y(t)} d u-\int_{0}^{T} e^{\tilde{Y}(u)-\tilde{Y}(t)} d u\right|^{p}\right)^{1 / p} \\
\leq & 2^{1 / p} \sqrt{v_{1}} A_{N, 0} p^{1 / 2} \exp \left\{2 p v_{2} B(0)-\frac{1}{2}\right\}
\end{aligned}
$$

where $A_{N, 0}=\left.A_{N, t}\right|_{t=0}$. The entropy integral appearing in Corollary 2.1 is estimated with the help of Lemma 2.2 .

$$
\int_{0}^{\theta \varepsilon_{0}}\left(\frac{T}{\varphi^{(-1)}(\varepsilon)}\right)^{1 / p} d \varepsilon \leq \int_{0}^{\theta \varepsilon_{0}}\left(T\left(\frac{G_{p}}{\varepsilon}\right)^{1 / \beta}\right)^{1 / p} d \varepsilon=\frac{\theta^{1-1 /(p \beta)} T^{\beta} G_{p}}{1-\frac{1}{p \beta}}
$$

for $1-(p \beta)^{-1}>0$ and $G_{p}=\left.G_{p, t}\right|_{t=T}$. Since the function

$$
f(\theta)=\frac{1}{\theta^{1 /(p \beta)}(1-\theta)}
$$

attains its minimum value at the point $\theta_{0}=(p \beta+1)^{-1}$ if

$$
\theta_{0}<\frac{\varphi(T / 2)}{\varepsilon_{0}},
$$

we obtain, after simple algebra, that

$$
\inf _{0<\theta<\varphi(T / 2) / \varepsilon_{0}} \frac{1}{\theta^{1 /(p \beta)}(1-\theta)} \frac{T^{\beta} G_{p}}{1-(p \beta)^{-1}} \leq T^{\beta} G_{p} \frac{(p \beta+1)^{1+1 /(p \beta)}}{p \beta-1} .
$$

Taking into account (16) and the inequality $(a+b)^{p} \leq 2^{p-1}\left(a^{p}+b^{p}\right)$, we get from Corollary 2.1

$$
\begin{aligned}
\mathrm{P}\left\{\sup _{0 \leq t \leq T}\left|\int_{0}^{T} e^{Y(u)-Y(t)} d u-\int_{0}^{T} e^{\tilde{Y}(u)-\tilde{Y}(t)} d u\right|>\alpha\right\} \\
\quad \leq \frac{2^{p-1}\left(2^{1 / p} \sqrt{v_{1}} A_{N, 0} p^{1 / 2} \exp \left\{2 p v_{2} B(0)-\frac{1}{2}\right\}\right)^{p}}{\alpha^{p}}+\frac{2^{p-1}\left(T^{\beta} G_{p} \frac{(p \beta+1)^{1+1 /(p \beta)}}{(p \beta-1)}\right)^{p}}{\alpha^{p}} .
\end{aligned}
$$

The definition of $G_{p}$ and the inequality

$$
\frac{p^{p}}{(p \beta-1)^{p}} \leq \frac{1}{\beta^{p}} \exp \left\{\frac{p}{p \beta-1}\right\}
$$

imply

$$
\begin{aligned}
& \mathrm{P}\left\{\sup _{0 \leq t \leq T}\left|\int_{0}^{T} e^{Y(u)-Y(t)} d u-\int_{0}^{T} e^{\tilde{Y}(u)-\widetilde{Y}(t)} d u\right|>\alpha\right\} \\
& \quad \leq \frac{L_{N}^{p} p^{p / 2} \exp \left\{2 p^{2} v_{2} B(0)-p / 2\right\}}{\alpha^{p}}+\frac{M_{N}^{p} e^{p /(p \beta-1)}(p \beta+1)^{p+1 / \beta} e^{16 p^{2} B(0)-p / 2}}{\sqrt{2} \alpha^{p}}
\end{aligned}
$$


where

$$
L_{N}=2 \sqrt{v_{1}} A_{N, 0}, \quad M_{N}=\frac{2 T^{\beta}}{\beta}\left(T \sqrt{2 P_{N}}+\left(2^{7-2 \beta} \Lambda^{2 \beta} B(0)\right)^{1 / 2} A_{N, T}\right) .
$$

Evaluating the right-hand side at the point

$$
p_{0}=\frac{\frac{1}{2}-\ln M_{N}}{32 B(0)},
$$

which is close to the point of minimum of the function

$$
\frac{M_{N}^{p} e^{p /(p \beta-1)}(p \beta+1)^{p+1 / \beta} e^{16 p^{2} B(0)-p / 2}}{\sqrt{2} \alpha^{p}},
$$

we prove bound (15), since the condition $1-(p \beta)^{-1}>0$ implies $\theta_{0}<\varphi(T / 2) / \varepsilon_{0}$ by Corollary 2.1. This completes the proof of Lemma 2.3.

Theorem 2.2. Let $Y(t)$ be a separable centered mean square continuous stationary Gaussian stochastic process belonging to the space $\mathrm{L}_{p}(\Omega), p \geq 1$, which means that

$$
\mathrm{E}|Y(t)|^{p}<\infty \quad \text { for all } t \in T \text {. }
$$

Denote the spectral function of the process $Y$ by $F(\lambda)$. Assume that the spectral moment

$$
\int_{0}^{\infty} \lambda^{2 \beta} d F(\lambda), \quad 0<\beta \leq 1,
$$

exists. Let a partition $D_{\Lambda}$ of the interval $[0, \Lambda], \Lambda \in \mathbf{R}$, be such that $\lambda_{k-1}-\lambda_{k}=\Lambda / N$, $N \in \mathbf{N}$. Then a model $\{\widetilde{\nu}(B), B \in \mathfrak{B}\}$ of a log Gaussian Cox process, where $\widetilde{Y}(t)$ is defined by (11), approximates the process with accuracy $\alpha$ and reliability $1-\gamma$ if

$$
\begin{gathered}
M_{N}<\alpha \exp \left\{\frac{1}{2}-\frac{32 B(0)}{\beta}\right\}, \\
\left(\frac{L_{N}}{\alpha}\right)^{K_{N}} K_{N}^{K_{N} / 2} \exp \left\{-\frac{K_{N}}{2}+2 v_{2} K_{N}^{2}\right\} \\
+\frac{1}{\sqrt{2}} \exp \left\{\frac{K_{N} \beta}{K_{N} \beta-1}\right\}\left(K_{N} \beta+1\right)^{K_{N}+1 / \beta} \exp \left\{-16 B(0) K_{N}^{2}\right\} \leq \gamma,
\end{gathered}
$$

where the constants $M_{N}$ and $K_{N}$ are defined in Lemma 2.3.

Proof. Theorem 2.2 follows from Definition 2.1 and Lemma 2.3 .

\section{COnCluding REMARKS}

A method for modelling Cox processes is proposed in this paper for the case where the intensity is generated by a Gaussian process. The method is based on the spectral decomposition of the process generating the intensity and can be generalized to the case of non-Gaussian stochastic processes that admit a spectral representation. The models described in the paper are constructed with a given accuracy and reliability.

\section{BIBLIOGRAPHY}

1. P. J. Diggle, Statistical Analysis of Spatial Point Patterns, Academic Press, London, 1983. MR.743593 (85m:62205)

2. N. Cressie, Statistics for Spatial Data, Wiley, New York, 1991. MR1127423 (92k:62166)

3. J. Møller, A. R. Syversveen, and R. P. Waagepetersen, Log Gaussian Cox processes, Scand. J. Statist. 25 (1998), no. 4, 451-482. MR.1650019 (2000k:62156)

4. J. Møller and R. P. Waagepetersen, Statistical Inference and Simulation for Spatial Point Processes, Chapman \& Hall/CRC, Boca Raton, FL, 2004. MR2004226 (2004h:62003) 
5. J. Møller, Spatial Statistics and Computational Methods, Springer-Verlag, New York, 2003. MR2001383 (2004f:62012)

6. O. O. Pogorilyak, Modeling log Gaussian Cox processes, Visnyk Kyiv Taras Shevchenko National University, Ser. Matem. Mekh. 2006, no. 15-16, 94-100. (Ukrainian)

7. Yu. V. Kozachenko and O. O. Pogorilyak, Modeling log Gaussian Cox processes with given reliability and accuracy, Teor. İmovir. Matem. Statyst. 76 (2007), 70-83; English transl. in Theory Probab. Math. Statist. 76 (2008), 77-91. MR.2368741

8. Yu. V. Kozachenko and O. O. Pogorilyak, Modeling log Cox processes governed by a random field, Dopovidi NAN Ukrainy 2006, no. 10, 20-23. (Ukrainian)

9. V. V. Buldygin and Yu. V. Kozachenko, Metric Characterization of Random Variables and Random Processes, Amer. Math. Soc., Providence, Rhode Island, 2000. MR.1743716 (2001g:60089)

10. H. Cramér and M. R. Leadbetter, Stationary and Related Stochastic Processes. Sample Function Properties and their Applications, John Wiley \& Sons, New York-London-Sydney, 1967. MR.0217860(36:949)

Department of Probability Theory and Mathematical Statistics, Faculty for Mechanics and Mathematics, National Taras Shevchenko University, Academician Glushkov Avenue 6 , KYIV 03127, UKRAINE

E-mail address: yvk@univ.kiev.ua

Department of Probability Theory and Mathematical Statistics, Faculty for Mechanics and Mathematics, National Taras Shevchenko University, Academician Glushkov Avenue 6 , KYIV 03127, UkRAINE

E-mail address: alex_pogorilyak@ukr.net

Received 26/DEC/2006

Translated by OLEG KLESOV 